\title{
Technology and applications of micromachined silicon adaptive mirrors
}

\author{
Gleb Vdovin \\ Simon Middelhoek \\ Delft University of Technology \\ Laboratory of Electronic Instrumentation \\ P.O. Box 5031 \\ 2600 GA, Delft, The Netherlands \\ E-mail: gleb@ei.et.tudelft.nl \\ Pasqualina M. Sarro \\ Delft University of Technology \\ DIMES \\ P.O. Box 5053 \\ 2600 GB, Delft, The Netherlands
}

\begin{abstract}
The technology of low-cost high-quality micromachined adaptive mirrors is reported. Adaptive mirrors are fabricated by combining bulk silicon micromachining with standard electronics technologies. Mirrors with tens of control channels, having RMS initial deviation from plane of the order of $\lambda / 20$ and a range of surface deflection of 10 to 20 $\mu \mathrm{m}$ with linear frequency response in the range of $50 \mathrm{~Hz}$ to $1 \mathrm{kHz}$, are fabricated on standard PCB substrates. Advanced devices with hundreds of control channels, demanding integration of driver and switching electronics, are currently under development. (c) 1997 Society of Photo-Optical Instrumentation Engineers. [S0091-3286(97)00605-3]
\end{abstract}

Subject terms: micro-opto-electro-mechanical systems; micromachining; adaptive optics; adaptive mirror; wavefront control; zoom lens.

Paper MEM-06 received Oct. 14, 1996; revised manuscript received Jan. 8, 1997; accepted for publication Jan. 16, 1997.

\section{Introduction}

Adaptive optics ${ }^{1,2}$ as a technology combines optics, mechanics, and electronics and puts a high demand on additional investment into specialized know-how as well as a highly skilled workforce. Adaptive optical systems are complex and expensive because no uniform technology exists for the fabrication of adaptive mirrors, control systems, and wavefront sensors. For example, the high-quality membrane adaptive mirror reported in Ref. 3 was fabricated with a complex technology, which evidently could not be transferred into mass production. So far, the technology has been developed in the direction of improving the system quality, with very little attention given to system costs. Expensive adaptive systems, as they are, are suitable for special applications such as ground-based telescopes and military equipment. Further extension of adaptive optics into fields of mass-produced optical systems and optoelectronics can only be possible along with the reduction of fabrication costs by a few orders of magnitude. This transition must preserve, as much as possible, the quality standards of more expensive systems. As the market for low-end optoelectronics such as CD-player optical pickups, guidance systems for amateur telescopes, optical communication systems, displays, and photo and video lenses is comparable to or wider than the aforementioned high-end military and scientific markets, serial or even mass-production technologies should be developed for the fabrication of high-quality adaptive optical devices.

Silicon micromachining ${ }^{4,5}$ has recently been successfully applied to the fabrication of optical devices, such as deformable mirrors and displays. ${ }^{6,7}$ Micromachined adaptive mirrors ${ }^{8,9}$ combine low cost and simplicity of control with the possibility of mass production. Because of these features, whole new fields of applications have become possible.
In the present article the authors report their recent results in the development of micromachined adaptive mirrors.

\section{General Principles}

New-generation adaptive mirrors must provide the functionality of expensive high-end devices at a low cost. Thus the technology should be transferable to mass production, allowing for uniform fabrication of optical surfaces, actuators, and control electronics. Bulk silicon micromachining is suitable for this purpose, as it is supported by many silicon IC industries. Fabrication of membrane adaptive mirrors ${ }^{10}$ with bulk micromachining provides the following advantages in the fields of optics, electrical actuation and control electronics:

- Bulk micromachining allows for the fabrication of thin flexible membranes with high optical quality, because released membranes replicate the surface of the polished wafer. These membranes are optically plane because they are fabricated tensile prestressed. Tens of membranes can be fabricated on one silicon wafer with a high yield.

- The shape of a reflective membrane can be controlled electrostatically by applying voltages between the membrane and the array of electrostatic actuators. This is a natural means of actuation, providing functionality for a very low cost.

- Combining micromachining with standard IC technology, mirror drivers and control electronics can be integrated into the adaptive mirror substrate together with the actuator structure. This can greatly reduce the system complexity, allowing for implementation of more sophisticated control algorithms.

Implemented membrane mirrors are formed by thin films stretched over windows etched in silicon chips. These 


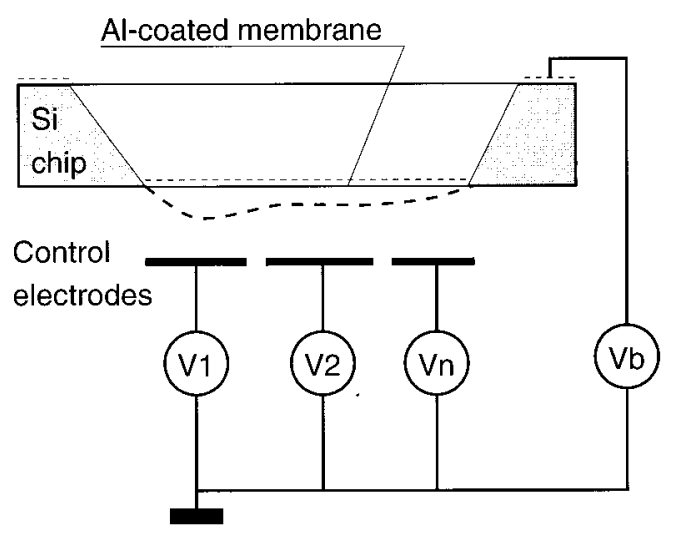

Fig. 1 Principle of micromachined adaptive mirror.

membranes are coated appropriately for high reflectivity and mounted over integrated electrode structures. Cost, application, and performance of fabricated devices depend on the design and technology used. These aspects are considered in more detail in the following section.

\section{Fabrication Technology}

A typical micromachined adaptive mirror, shown schematically in Fig. 1, consists of two parts: the chip with a flexible reflective membrane, and the structure with control electrodes.

The membrane is formed by low-stress low-pressure chemical-vapor-deposited (LPCVD) silicon nitride with a thickness in the range 0.3 to $0.8 \mu \mathrm{m}$. Such a membrane can withstand tens of billions of deflection cycles without changing its tension and initial surface quality. Fabrication of membranes is carried out on standard equipment that provides precise control over the membrane stress, important for fabrication of devices with predictable sensitivity and frequency characteristics.

To fabricate the membrane, both sides of a silicon wafer are coated with layers of nitride. During the next step, windows are open by dry-etch patterning the nitride on the back side of the wafer. Bulk silicon is etched through these windows, by submerging the wafer in $33 \%$ water solution of $\mathrm{KOH}$ at $85^{\circ} \mathrm{C}$. This solution does not etch nitride; therefore, etching results in thin nitride membranes, covering the windows etched in the silicon wafer. Ten to twenty membranes with a characteristic size of $1 \mathrm{~cm}^{2}$ can be fabricated on a standard 4-in. wafer.

The response function of a membrane adaptive mirror depends on the shape of the etched window. Special technologies should be used to fabricate different window geometries. The main complication is introduced by the fact that anisotropic etching of $\langle 100\rangle$ monocrystalline silicon wafers in $\mathrm{KOH}$, traditionally used in bulk micromachining, produces rectangular windows regardless of the etchingmask shape-see Fig. 2. Isotropic etching, which can be conducted, for example, in a mixture of $\mathrm{HF}$ and $\mathrm{HNO}_{3}$ at room temperature, produces very smooth rounded contours, which repeat approximately the shape of the mask with an achievable lateral resolution comparable to the wafer thickness. The isotropic etchant dissolves the nitride and should be handled with great care, as it etches the majority of

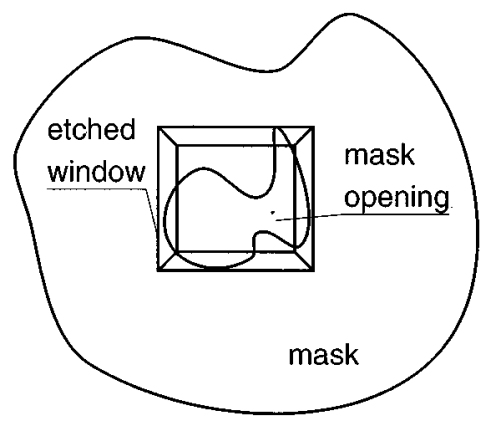

Fig. 2 General rule for anisotropic etching: Any etching mask results in a rectangular opening.

construction materials and is very poisonous. To control the window (membrane) shape precisely, avoiding as much as possible the use of isotropic etchants, special compensation methods should be applied in the anisotropic etching step. Compensation results in a better approximation to the desired window shape, formed by sharp cornerlike crystalline silicon structures. To improve the mechanical reliability of the membrane, the processing should be finished with a short isotropic etching step to dissolve these sharp imperfections. This step results in a smoothing of the contours and a reduction in the dynamically induced local stresses during membrane deformation.

The etched side of the membrane, replicating the surface of a polished silicon wafer, is usually coated with a reflective aluminum layer. A planar electrode structure can be placed very close to the other uncoated side of the membrane, to achieve membrane deflections under moderate control voltages.

The distance between the membrane and the electrode structure is controlled by a dielectric spacer, supporting the membrane die. Different technologies can be used for fabrication of the spacers and control electrodes. In the simplest case, electrodes are formed in the metallization layer directly on the surface of a PCB holder. Lateral resolution of the structures patterned in the PCB metallization layer is limited to 20 to $100 \mu \mathrm{m}$, making the PCB technology ideally suitable for low-resolution adaptive mirrors with 1 to 100 actuators. The thicknesses of different PCB layers such as the metallization layer and the antisoldering mask are well defined; therefore one of these layers can be used as a spacer, to define the distance between the mirror and the electrode structure. For example, if the metallization has a thickness of $35 \mu \mathrm{m}$ and the antisoldering layer has a thickness of $75 \mu \mathrm{m}$, then three small antisoldering islands, patterned directly onto PCB surface, will support the chip with the mirror membrane at a distance of $40 \mu \mathrm{m}$ over the electrodes. The same islands placed over the metallization will define a distance of $75 \mu \mathrm{m}$. Support at three points introduces no additional stress into the mirror die, preserving the planarity of the stretched membrane.

High-resolution electrode patterns with hundreds of electrodes can be fabricated with standard IC technologies in silicon. In the simplest case, a silicon die is coated with a thick layer (a few micrometers) of oxide, over which the aluminum electrodes are patterned with a typical resolution of $2 \mu \mathrm{m}$. Examples of silicon-based electrode structures are shown in Fig. 4. This technology is more complicated with 


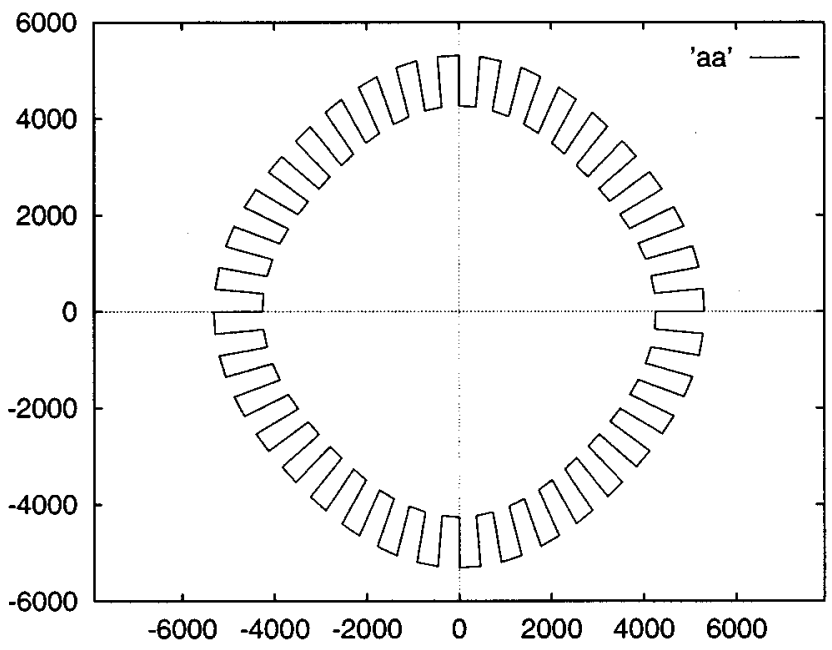

Fig. 3 Geometry of the etch mask to obtain a circular membrane using $\mathrm{KOH}$ anisotropic etching. All sizes are in micrometers.

respect to assembly and alignment of mirror components. An external spacers should be placed between the membrane die and the electrode structure to define the distance. The dies are glued together and the spacer is removed, to reduce stress and deformation in the mirror. A further increase in the number of electrodes can be achieved by the integration of driver, switching, and addressing electronics into a single device.

\section{Etching Compensation}

In the case of membrane deflection induced by a single electrode providing uniform electrostatic pressure over the whole surface, the optical figure of the deformed membrane depends on the contour shape. Square contours produce cushionlike responses, circular contours produce perfect parabolic responses, elliptic contours produce astigmatic surfaces, and contours in the form of a long slit produce almost perfect cylindrical shapes in their central part.

Fabrication of square and rectangular windows is achieved by anisotropic etching of $\langle 100\rangle$ wafers-see Fig. 2 . A special etch mask with a negative corner compensation must be applied (see Fig. 3) to obtain a membrane with an approximately circular aperture using standard $\mathrm{KOH}$ anisotropic etching. This compensation results in an approximately circular membrane with a diameter of 8 to $12 \mathrm{~mm}$, deviating from the circular contour by less than $0.2 \mathrm{~mm}$. This deviation does not depend on the diameter of the membrane, so larger circular membranes can be fabricated with a relatively better approximation. An example of an approximately circular opening etched anisotropically in a silicon chip is shown in Fig. 4.

Apertures etched anisotropically with compensation display small stepwise deviations from the circle-see Fig. 4. When a membrane, stretched on a contour with radius $a$, is deformed by a constant pressure, the deviation from the nearest parabola, with a radius of curvature $R$, due to the error $\delta a$ in the contour shape, is given by the formula

$\epsilon=a \delta a / R$

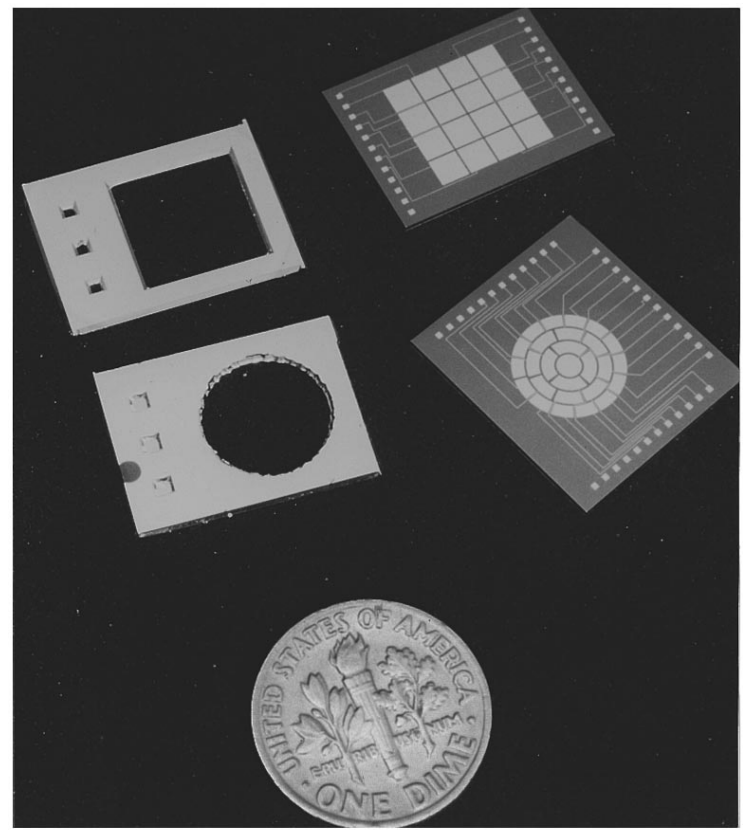

Fig. 4 Circular and rectangular apertures, etched anisotropically in silicon chips (left), and the corresponding silicon-based actuator structures (right).

or

$\epsilon / \delta a=a / R$.

From the latter expression we see that the demands on the shape of the membrane contour are very strict. For example, to achieve $\epsilon=0.1 \mu \mathrm{m}$ with a membrane mirror having a diameter of $1 \mathrm{~cm}$, when the deformed membrane has a focal distance of $1 \mathrm{~m}$, the deviation from the circular contour must be less than $20 \mu \mathrm{m}$.

The compensation reported can be applied to the fabrication of more complex shapes, for example for elliptical mirrors to correct astigmatic beams emitted by semiconductor lasers.

\section{Defocus Correctors}

Safe and inexpensive micromachining of approximately circular nitride membranes facilitates the fabrication of varifocal mirrors. In such a mirror the reflective membrane is deflected by a single electrode to provide the possibility of fast control of the optical power in the range of a few diopters. The optical quality, speed of response, aberrations, and sensitivity of such a device are functions of the design parameters and the technology used.

The most common case is represented by a circular nitride membrane, fabricated with anisotropic etching and suspended 50 to $100 \mu \mathrm{m}$ over a conductive substrate, such as a silicon chip or a PCB.

The assembled circular membrane mirror, consisting of a 10-mm-diam membrane mounted $75 \mu \mathrm{m}$ above a single control electrode, is shown in Fig. 5. Typical interferograms of the initial and deformed mirror surface are shown in Fig. 6.

There are three main sources of aberration in integrated defocus correctors: 


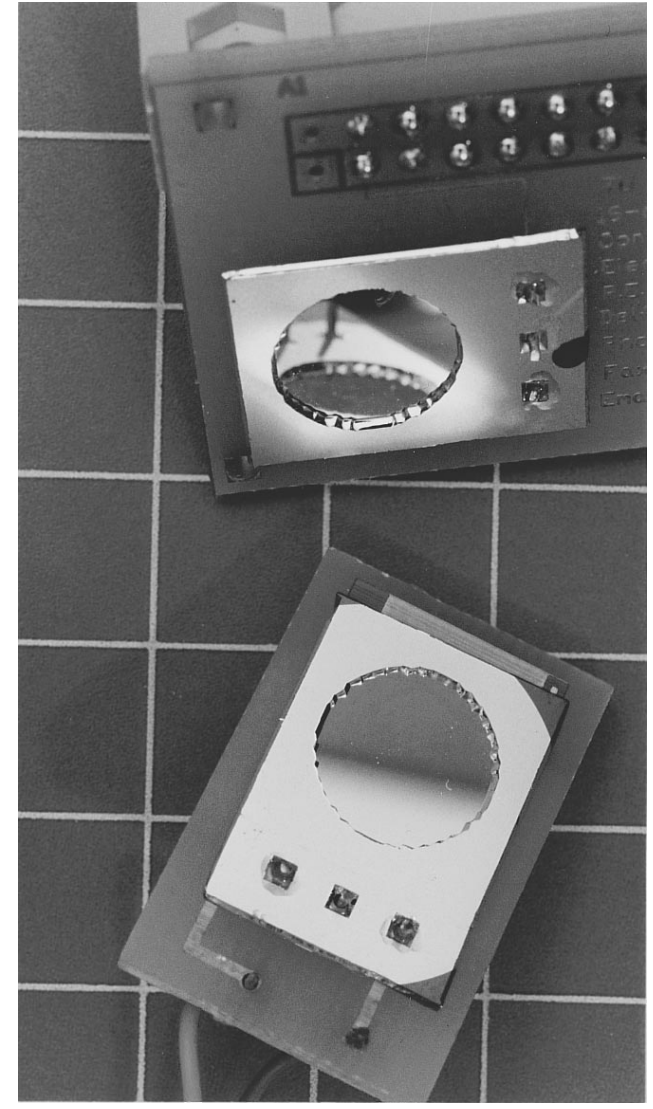

Fig. 5 PCB-based assembled adaptive mirrors: circular controlled by a 19-channel electrode structure (top) and a single-channel defocus corrector (bottom).

- Spherical aberration, caused by the nonlinearity of the force field between the actuator and the deformed membrane. The central part of the deflected membrane has a higher curvature because it is subjected to higher electrostatic pressure. The pressure nonuniformity may reach $10 \%$ to $20 \%$ in a typical case with a mirror having a diameter of $1 \mathrm{~cm}$, a gap thickness of $70 \mu \mathrm{m}$, and a focal-distance range from $\infty$ to $1 \mathrm{~m}$. The local mirror curvature is proportional to the pressure applied; therefore, the curvature nonuniformity can also reach $10 \%$ to $20 \%$.

- Coma-like aberration due to membrane misalignment with respect to the electrode. It was shown experimentally that a gap nonuniformity of $1.5 \%$ in a mirror with an 8 -mm light diameter reduces the Strehl ratio to a value of 0.1 at a focal distance of $1 \mathrm{~m}$.

- Initial astigmatism of the membrane.

Spherical aberration of a membrane defocus corrector can be reduced by increasing the gap between the electrode and the membrane. The control voltage required is proportional to the square of the gap thickness; therefore this method is not very useful in practice, because a linearized mirror will require control voltages of thousands of volts. Spherical aberration can also be reduced by using the actuator structure, consisting of several concentric rings. As a rough approximation, the voltage applied to each of these
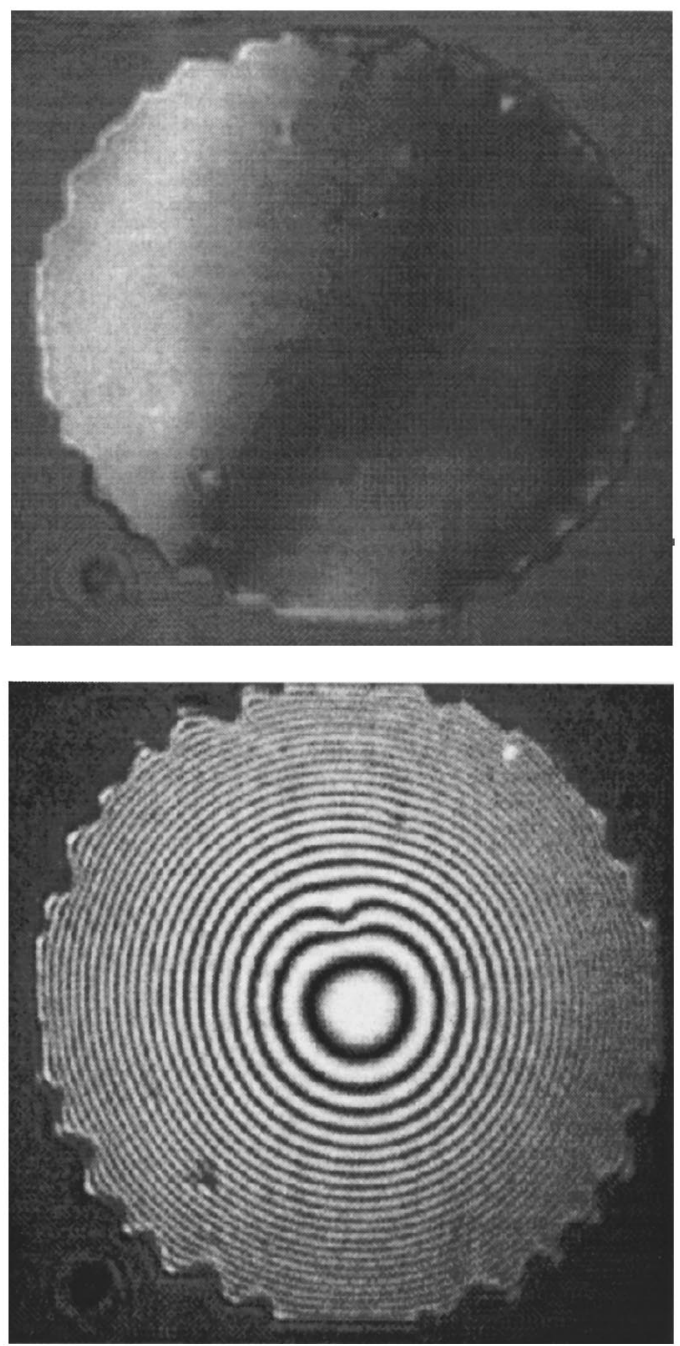

Fig. 6 Interferogram of the initial mirror surface with an active diameter of $10 \mathrm{~mm}$ (top); the same deformed by the control voltage of 90 $\mathrm{V}$, which corresponds to a focal distance of $1 \mathrm{~m}$ (bottom).

rings must be inversely proportional to the square of the average distance between the chosen actuator and the deformed membrane, to achieve a uniform distribution of the membrane curvature. Computer simulation of this situation and some practical recommendations can be found in Ref. 11. In the practical situation, control with axisymmetric annular electrode structures should be avoided, as it provides no means for compensation of aberrations caused by membrane decentering and misalignment.

Aberrations due to membrane misalignment can be reduced by fabricating the spacer and actuator structures in a single technological process. Mounting over PCB holders with spacers patterned in the antisoldering layer provides a better gap uniformity than mounting over silicon structures using an external spacer. Further adjustments can be achieved with simple $2 \times 2$ square actuator patterns. Individual actuator voltages can be preadjusted using a set of voltage dividers so that the whole device can still be controlled by a single external voltage.

The dynamics of response of the defocus corrector de- 
pends on the membrane resonant frequency and the air damping. Usually the air damping limits the cutoff frequency to between 50 and $1000 \mathrm{~Hz},{ }^{9}$ which is much lower than the resonant frequency of the membrane. The damping depends on the ambient gas pressure, the distance between the membrane and the electrode structure, and the amplitude of membrane oscillations. Frequency responses for small and large signals are different. Perforation of the actuator structure, facilitating air flow under the membrane, reduces the air damping, thereby increasing the cutoff frequency. For example, a defocus corrector with a diameter of $1 \mathrm{~cm}$ and an amplitude of optical power of 3 diopters features a linear frequency response in the range 0 to 75 Hz. This means that the component can be switched between infinity and a focal distance of $0.3 \mathrm{~m}$ with a frequency of at least 25 cycles per second.

The PCB-mounted micromachined varifocal mirrors are simple, inexpensive, fast, and reliable. A typical singleelectrode corrector, with a usable light aperture of 6 to 9 $\mathrm{mm}$, shows reasonable optical quality (with peak-valley deviation from the reference parabola less than one wavelength) in a range of focal distances from $\infty$ to $50 \mathrm{~cm}$ under a control voltage in the range 0 to $200 \mathrm{~V}$. The optical quality of the mirror can be further improved by using more complex actuator patterns to correct intrinsic aberrations.

\section{Focusing of Laser Beams}

Optical systems with apertures of the order of few millimeters are finding more and more applications because of the general trend of miniaturization in optics. Adaptive mirrors with light apertures of between 1 and $4 \mathrm{~mm}$ are applicable to these systems. For instance, laser beam diameters in the majority of consumer and industrial laser optical pickup systems do not exceed a few millimeters. Micromachined adaptive mirrors can be used in these devices for fast precision beam focusing.

There are two possible geometries of a flexible nitride membrane, applicable to laser beam focusing: circular and rectangular. As shown above, the circular membrane provides almost ideal focusing. On the other hand, methods of etch compensation, described above, are not applicable for small membranes, as the error of contour approximation becomes too large compared to the membrane size.

It was shown experimentally that a corrector formed by a rectangular $4 \times 4-\mathrm{mm}$ Al-coated nitride membrane suspended at a gap of 20 to $40 \mu \mathrm{m}$ over a single metal electrode provides focusing of laser beams with diameters up to $3 \mathrm{~mm}$ in the range $\infty$ to $10 \mathrm{~cm}$ with a Strehl parameter better than 0.2 .

The response of the membrane also depends on the shape of the control electrode. For a control electrode covering the whole membrane, the cushionlike response of the square membrane provides a good enough approximation to the parabolic shape when the deflection of the membrane does not exceed a few wavelengths and the light beam covers only the central part of the membrane. These conditions are usually satisfied in the case of laser beams focusing by a small rectangular membrane mirror.

A small electrode placed under the membrane center produces a conical mirror shape, which is close to the shape of a logarithmic axicon, the properties of which are de-
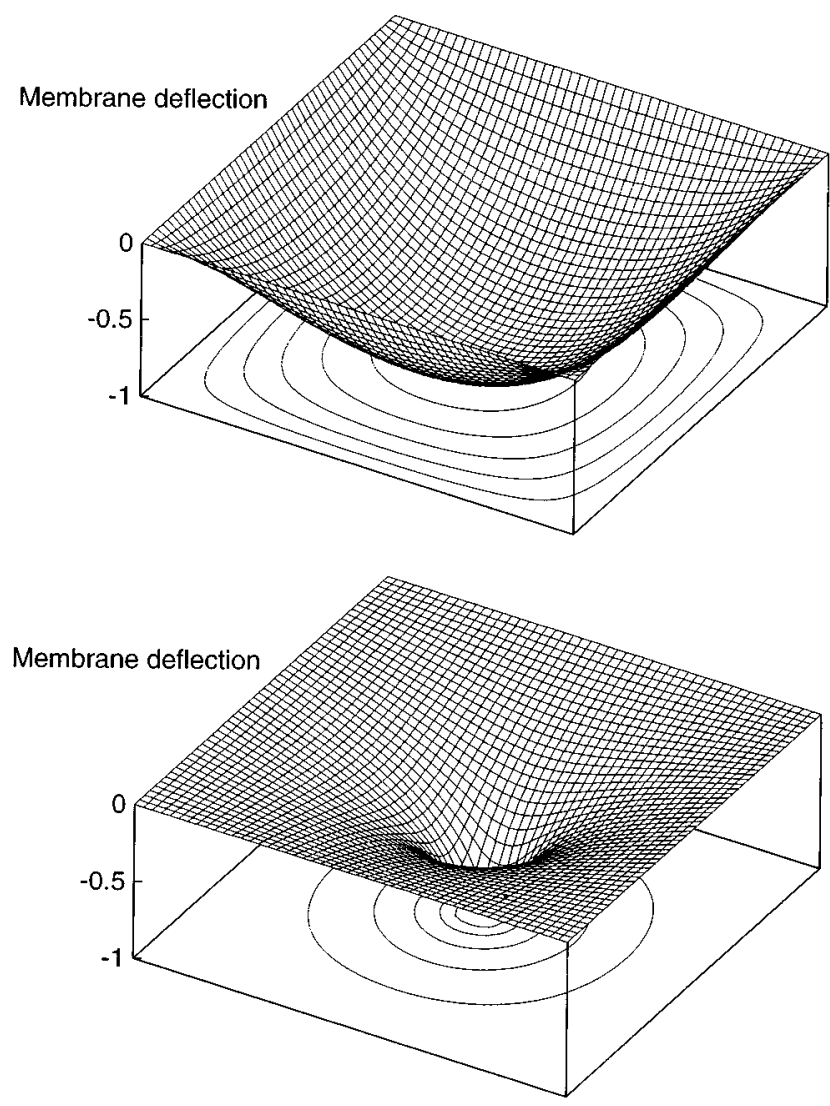

Fig. 7 Shape of a rectangular membrane deformed by an electrode covering the whole membrane is close to parabolic (top). The same membrane, deformed by a small central electrode, takes on an axicon-like shape (bottom).

scribed in Ref. 12. This shape produces a very long depth of focus with uniform focal intensity. Optical figures of the deformed membrane, obtained with large and small centered control electrodes, are compared in Fig. 7.

Quasiparabolic deformation by a large electrode produces a sharp and bright focal point with a small depth of focus. The focal distribution is comparable with that produced by an ideal lens-see Fig. 8. Axicon-like deformation produces a long uniform focal distribution. The example shown in Fig. 9 corresponds to the case of a Gaussian beam with $\lambda=650 \mathrm{~nm}$ focused by a rectangular $4 \times 4$-mm membrane deflected to an amplitude of $5 \mu \mathrm{m}$ by a centered electrode $0.5 \mathrm{~mm}$ in diameter. This distribution can be used in applications where illumination with a large depth of focus is demanded.

We have briefly considered only two limiting cases of a laser focusing device, controlled by a single electrode: the quasiparabolic focusator and the axicon-like focusator. Optimization of the electrode geometry facilitates control over the focal depths, so the whole range of focal distributions with different ratios of focal depth to the maximum axial intensity can be obtained with this simple and inexpensive device. 


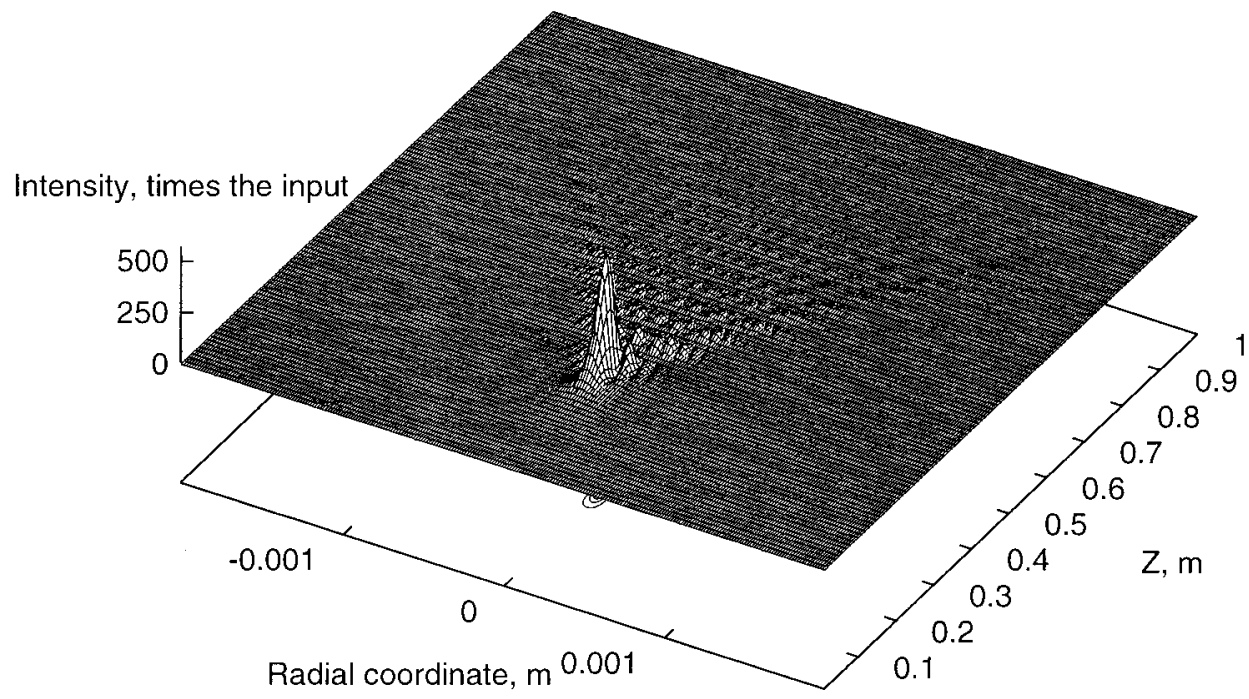

(a)

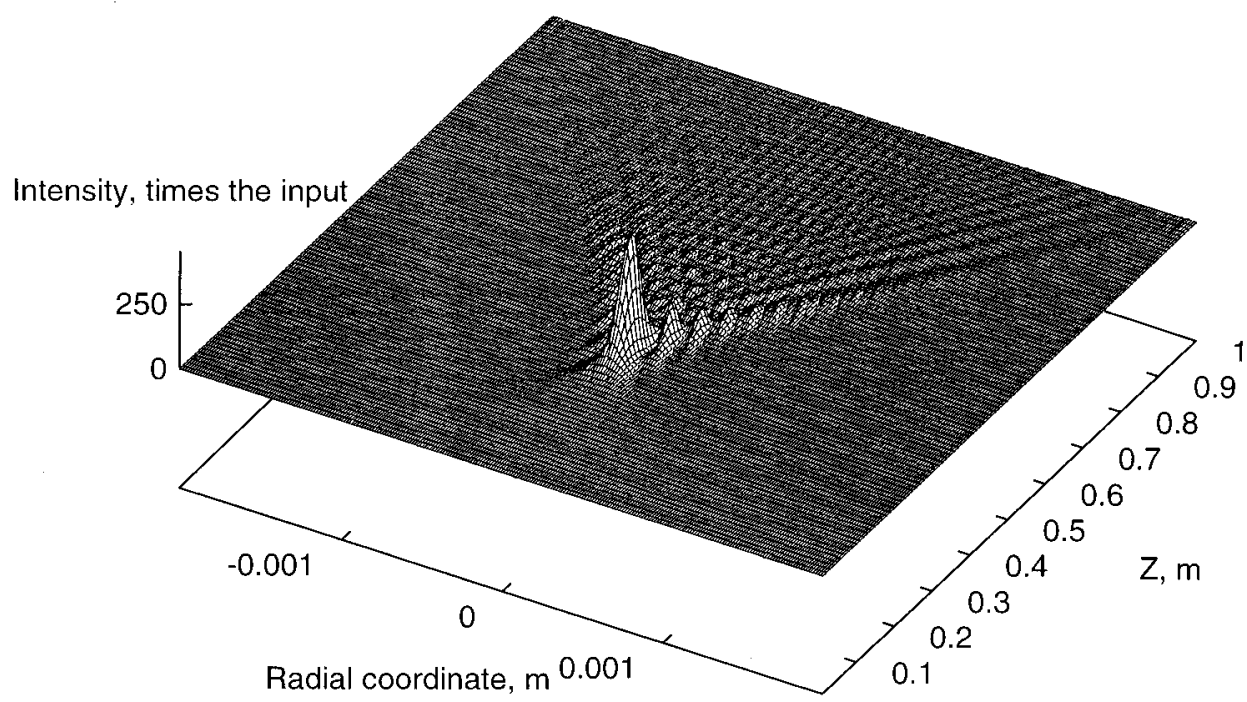

(b)

Fig. 8 Radial intensity distribution in Gaussian beam with $w_{0}=3 \mathrm{~mm}$, propagating after focusing by an ideal lens (a) with focal distance of $25 \mathrm{~cm}$ and a rectangular $4 \times 4-\mathrm{mm}$ membrane, deformed by a large electrode to equivalent focal distance (b).

\section{Multichannel Adaptive Mirrors}

The technology and applications of rectangular multichannel adaptive mirrors have been reported in Refs. 9, 10. Here we briefly report on results of two recent technological improvements:

- Fabrication of the mirror substrate, actuator structure spacers, and connectors using uniform PCB-based technology. The electrode structures patterned into a silicon chip are shown in Fig. 10. Silicon-based electrode structures require cumbersome and complicated assembly procedures. As a comparison, a PCB-based electrode structure with interconnects and connector pads is shown in Fig. 10. Its main advantage consists in the possibility of integrating all functional components onto the printed board.

- Development of the technology for the fabrication of comparatively large, approximately circular, nitride membranes.

These techniques have simplified the fabrication technology, making possible serial production of membrane adaptive mirrors in the price range of 10 to 100 U.S. dollars per control channel in a very careful estimation. An example of a PCB-based multichannel adaptive mirror is 


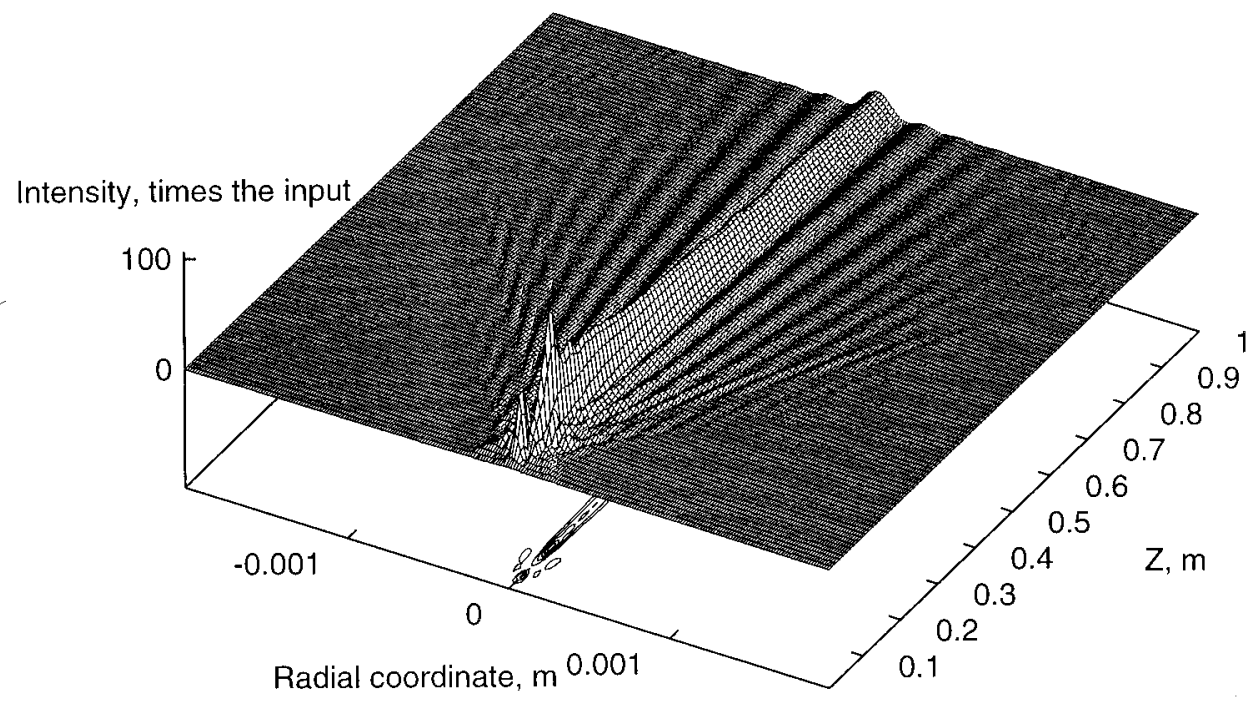

Fig. 9 Radial intensity distribution in a Gaussian beam, propagating after focusing by a $4 \times 4$-mm rectangular membrane, deformed by single electrode of diameter of $0.5 \mathrm{~mm}$ placed under the membrane center. The shape of the deformed membrane is illustrated in Fig. 7.

shown in Fig. 5. PCB-based mirrors have good optical quality, which is demonstrated by the interferograms shown in Fig 11.

In the standard case, electrostatic actuators are connected to conducting tracks on the back side of the PCB by means of vias (metallized holes). These holes reduce the air damping, extending the linear frequency response of the micromachined mirror to a few kilohertz, which is better

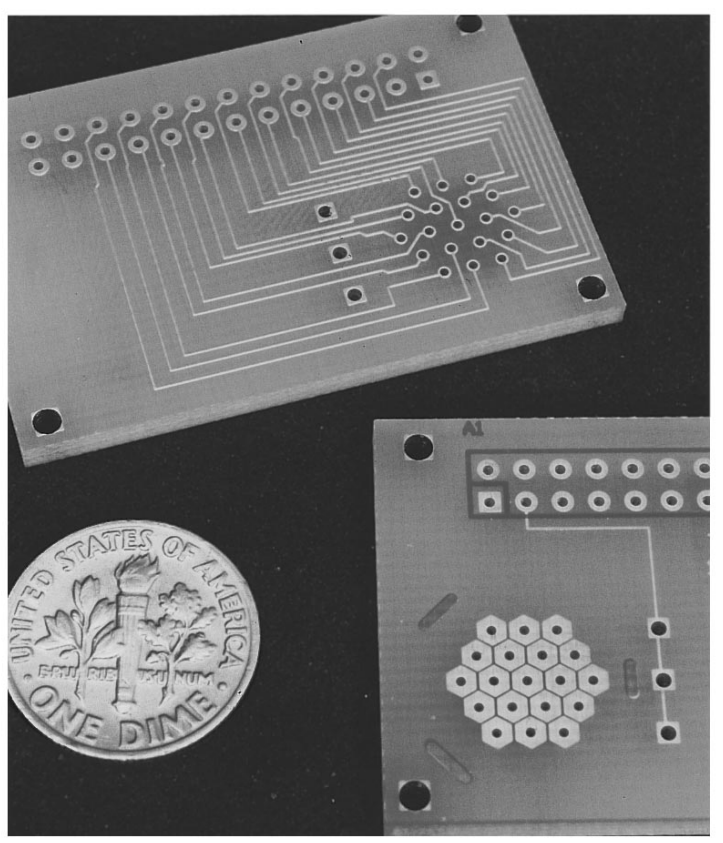

Fig. 10 Front and back sides of the PCB mirror substrate, including electrostatic actuators, electrode connections, contact pads for the band-cable connector, and the integrated spacer. than for similar devices mounted over planar silicon dies. ${ }^{9,11}$ Weak damping of the PCB-based mirrors increases the probability of excitation of higher-order mechanical harmonics, which might complicate the mirror control in high-frequency multichannel adaptive optical systems.

In the present design the reflective surface of micromachined mirror can be deflected only towards the actuators. To achieve bidirectional movement of the membrane, the mirror should be preliminarily biased electrically with a voltage $V_{b}$ as shown in Fig. 1. When all control voltages $V_{1}, \ldots, V_{n}$ are equal to zero, the membrane will take on a parabolic shape under the action of $V_{b}$. Application of bipolar control voltages to the biased device will provide bidirectional movement of the membrane. The voltage sensitivity is proportional to the bias voltage $V_{b}$. Bias voltages of the order of 50 to $200 \mathrm{~V}$ improve the mirror sensitivity so that control voltages of the order of 15 to $30 \mathrm{~V}$ are sufficient for many applications.

Multichannel adaptive mirrors can be used to form precision aspherical shapes, to correct aberrations of lowpower-laser beams, for image stabilization in astronomy and optical instrumentation, for use as low-resolution spatial light modulators, ${ }^{13}$ and finally for focusing photographic and video lenses. Anisotropic etch compensation and mounting on simple PCB carriers can significantly reduce the complexity of fabrication, providing inexpensive adaptive optics. The control electronics and the chip containing the flexible mirror can be placed on a single PCB, providing a somewhat old-fashioned but very functional integration. This simple technology is apparently not scalable to the devices having hundreds of control channels. These mirrors will require integration of the switching and driver electronics and the actuator structures in the common silicon substrate. The authors believe that developments in this direction can eventually lead to integration and standardiza- 

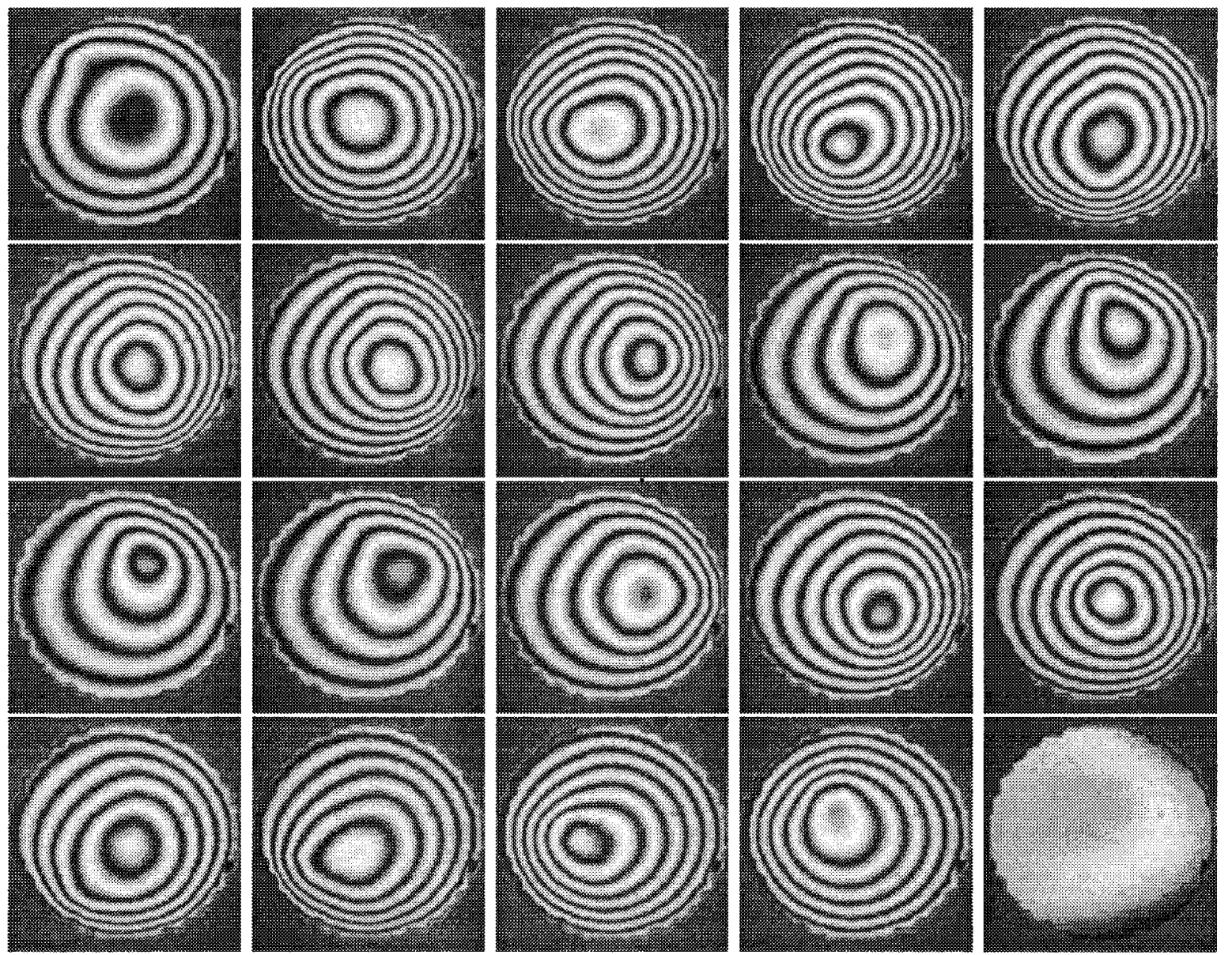

Fig. 11 The initial optical figure of the mirror (bottom right) and the response functions of 19-channel adaptive mirror mounted on the PCB shown in Fig. 10.

tion of the whole optoelectromechanical functional blocks of adaptive optical systems, as happened with electronics and computers.

\section{Conclusions}

The reported technologies of inexpensive micromachined adaptive mirrors have found many applications in modern optics and optoelectronics. Techniques developed for inexpensive fabrication of simple high-quality electrically controlled focus adjustment devices and correctors of special aberrations are currently being transferred into industry. In these devices, usually controlled by a single voltage, the optical figure demanded is achieved by combining special configurations of the membrane and the control electrode.

More complex devices, controlled by tens of actuators, are produced using standard PCB technology to fabricate the mirror carrier, actuator structure, spacer, electrical interconnects, and control electronics. These devices can replace more expensive adaptive optics in applications, where high costs prevent further development.

Finally, micromachined adaptive mirrors with hundreds of control channels are currently under development. These devices will achieve full integration of control and switching electronics, actuators, and flexible optical surfaces.

\section{Acknowledgments}

This work has been partly funded by a long-term ESPRIT project on micro-optical silicon systems (MOSIS).

\section{References}

1. R. K. Tyson, Principles of Adaptive Optics, Academic Press (1991).

2. M. A. Vorontsov, A. V. Koryabin, and V. I. Shmalgausen, Controllable Optical Systems (in Russian), Nauka (1988).

3. R. P. Grosso and M. Yellin, "The membrane mirror as an adaptive optical element,'” J. Opt. S. Am. 67, 399-406 (1977).

4. K. E. Petersen, "Silicon as a mechanical material," Proc. IEEE 70 , 420 (1982)

5. M. Mehregany, "Microelectromechanical systems," IEEE Circuits and Devices, pp. 14-22 (July 1993).

6. L. J. Hornbeck, "Projection displays and MEMS: timely convergence for a bright future," in Microelectronic Structures and Microelectromechanical Devices for Optical Processing and Multimedia Applications, Proc. SPIE 2641, 2 (1995).

7. M. Hisanaga, T. Koumura, and T. Nattori, "Fabrication of 3-dimensionally shaped Si diaphragm dynamic focusing mirror," in Proc. IEEE Workshop on MEMS, pp. 30-35 (1993).

8. L. M. Miller, M. L. Argonin, R. K. Bartman, W. J. Kaiser, T. W Kenny, R. L. Norton, and E. C. Vote, "Fabrication and characterization of a micromachined deformable mirror for adaptive optics applications,"' Proc. SPIE 1945, 421-430 (1993).

9. G. V. Vdovin and P. M. Sarro, "Flexible mirror micromachined in silicon," Appl. Opt. 34, 2968-2972 (1995).

10. G. V. Vdovin and P. M. Sarro, "Flexible reflecting membranes micromachined in silicon," Semiconductor Sci. and Technol. 9, 15701572 (1994)

11. G. V. Vdovin, S. Middelhoek, M. Bartek, P. M. Sarro, and D. Solo- 
matine, "Technology, characterization and applications of adaptive mirrors fabricated with IC-compatible micromachining," in Adaptive Optical Systems and Applications, Proc. SPIE 2534, 116-129 (1995).

12. J. Sochacki, Z. Jarosewicz, L. R. Staronski, and A. Kolodzeijchyk, “Annular-aperture logarithmic axicon,', J. Opt. Soc. Am. A10, 1765$1768(1993)$

13. G. V. Vdovin, "Spatial light modulator based on the control of the wavefront curvature," Opt. Comm. 115, 170-178 (1995).

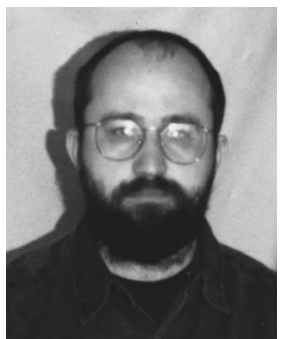

Gleb Vdovin received his engineering degree in optics from the Leningrad Institute of Fine Mechanics and Optics, USSR, in 1986. Since then he has been involved in laser and adaptive optics research with the Department of High-Power Lasers of the General Physics Institute of the Russian Academy of Sciences. In 1993 he joined the Electronic Instrumentation Laboratory of the Delft University of Technology, Netherlands. He received his $\mathrm{PhD}$ degree from the Delft University in 1996. His thesis dealt with silicon micromachined adaptive mirrors. His current research interests include adaptive optics, silicon microoptics, and displays. He is the author or co-author of more than 20 articles in the fields of laser and adaptive optics, optical sensors, and microoptics. Gleb Vdovin is a member of SPIE and OSA.

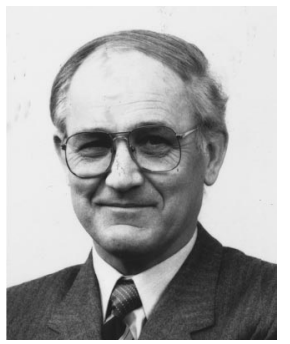

Simon Middelhoek received his applied physics engineering degree from Delft University of Technology, Delft, Netherlands, in 1956. He then joined the IBM Research Laboratory, Adliswil, Switzerland, as a research staff member, working on the physics of magnetic layers. In 1961, he obtained his $\mathrm{PhD}$ degree cum laude in mathematics and physics from the University of Amsterdam. From March 1962 to May 1963, he was with IBM Research in Yorktown Heights. He then rejoined IBM Research in Switzerland and developed the processing steps for the (at that time) fastest MESFET. In 1969 he joined the electrical engineering faculty of the Delft University of Technology and started a research program in the field of integrated silicon sensors. On October 1, 1996, he became professor emeritus and supervises $\mathrm{PhD}$ research projects on smart sensors for data acquisition. He has received the IBM Outstanding Contribution Award, several IBM patent awards, and the 1984 IEEE Centennial Medal. He is a fellow of the IEEE and member of the Royal Dutch Academy of Sciences and Foreign Associate of the NAE. He is general editor of the scientific journal Sensors and Actuators, which he inaugurated in 1981.

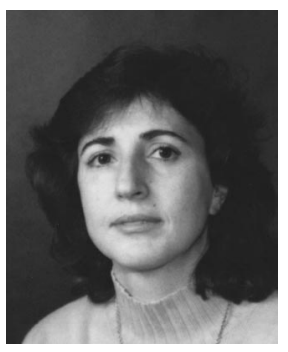

Pasqualina M. Sarro received her Laurea degree in solid-state physics from the University of Naples, Italy, in 1980. From 1981 to 1983 , show was a post-doctoral fellow in the Photovoltaic Research Group of the Division of Engineering, Brown University, Rhode Island, where she worked on thin-film photo-voltaic cell fabrication by chemical spray pyrolysis. In 1987, she received her $\mathrm{PhD}$ degree in electrical engineering from the Delft University of Technology, Netherlands. Her thesis dealt with infrared sensors based on integrated silicon thermopiles. Since then, she has been with the Delft Institute of Microelectronics and Submicron Technology (DIMES) at the Delft University, where she is responsible for research on integrated silicon sensors and microsystems technology. In April 1996 she became an associate professor in the Electronic Components, Materials, and Technology Laboratory of the Delft University. She has served as a technical program committee member of the ESSDERC '95, '96 and '97 Conferences for the subcommittee Sensors, Actuators, and Displays. 